\title{
Economic impact and effectiveness of radiation protection measures in aviation during a ground level enhancement
}

\author{
Daniel Matthiä ${ }^{1, *}$, Martin Schaefer ${ }^{2}$, and Matthias M. Meier ${ }^{1}$ \\ 1 German Aerospace Center, Institute of Aerospace Medicine, Linder Höhe, 51147 Cologne, Germany \\ ${ }^{*}$ Corresponding author: Daniel.Matthiae@dlr.de \\ 2 German Aerospace Center, Institute of Propulsion Technology, Linder Höhe, 51147 Cologne, Germany
}

Received 12 November 2014 / Accepted 8 May 2015

\begin{abstract}
In addition to the omnipresent irradiation from galactic cosmic rays (GCR) and their secondary products, passengers and aircraft crew may be exposed to radiation from solar cosmic rays during ground level enhancements (GLE). In general, lowering the flight altitude and changing the flight route to lower latitudes are procedures applicable to immediately reduce the radiation exposure at aviation altitudes. In practice, however, taking such action necessarily leads to modifications in the flight plan and the consequential, additional fuel consumption constrains the mitigating measures.

In this work we investigate in a case study of the ground level event of December 13th 2006 how potential mitigation procedures affect the total radiation exposure during a transatlantic flight from Seattle to Cologne taking into account constraints concerning fuel consumption and range.
\end{abstract}

Key words. Ground level enhancement - Solar energetic particles - Radiation protection - Aviation - Aircraft operations Monte-Carlo simulation - Radiation exposure - Effective dose - Ambient dose equivalent

\section{Introduction}

Radiation protection of aircrew members, who are occupationally exposed to cosmic rays at aviation altitudes, has been legally regulated in the EU since 1996 (EU 1996). An important principle in radiation protection is the so-called ALARAprinciple which demands that any unavoidable exposure to ionizing radiation should be kept as low as reasonably achievable, taking into account economic and societal factors (ICRP 2007). Since the radiation exposure increases with increasing height within the domain of civil aviation altitudes whereas fuel consumption generally decreases with increasing altitudes, there are conflicting interests concerning radiation exposure and economical flight operation. In consideration of the economic circumstances, operational flight plans are, by common consent in the aviation industry, optimized for operating costs only. With regard to the omnipresent radiation field at aviation altitudes, which is generated by galactic cosmic radiation, a reduction in flight altitude by $6000 \mathrm{ft}$ (ca. $1830 \mathrm{~m})$ would result in a decrease in dose rate of about $31 \%$ for polar routes and $24 \%$ for equatorial routes during the period of maximum exposure within the solar cycle, i.e. during solar minimum (Meier et al. 2009), while the fuel consumption would increase by about $8 \%$. During solar maximum conditions the ratio between reduction in dose rate and increase in fuel consumption would be even lower.

This situation can, however, change drastically during a Solar Particle Event (SPE) when, in rare cases, a huge amount of high-energy particles is expelled from the sun which can contribute significantly to the radiation field at aviation altitudes. Effects on the radiation exposure from solar energetic particles (SEP) usually last from a few minutes up to several hours. Since the dose rate gradient in dependence on altitude is usually much steeper for the solar particle contribution than for the galactic contribution of the radiation field due to the shape of the corresponding energy spectrum of the impinging particles, the ratio between reduction in dose rate and increase in fuel consumption for lower flight altitudes can become larger than for the galactic cosmic ray component alone. A solar particle event can last for several hours. This offers the temporary opportunity for more efficient mitigation measures based on the ALARA-principle of radiation protection.

A prerequisite for the effective implementation of mitigation measures is the timely availability of corresponding Space Weather information. For that purpose many airlines still rely on the NOAA $S$-scale, which is based on the integral proton flux above $10 \mathrm{MeV}$ outside the atmosphere. The integral flux, however, is dominated by protons with energies of a few tens of $\mathrm{MeV}$ which have no relevance for the radiation exposure at aviation altitudes. As a result, the $S$-scale is an inappropriate parameter for an assessment of the radiation exposure at aviation altitudes and consequently the Space Weather information given by the derived $S$-index is misleading for this scenario (Meier \& Matthiä 2014). A relevant parameter for such an assessment is given by the dose rate due to solar protons which provides the basis for an assessment of the Space Weather index $D$ (Meier \& Matthiä 2014) as a proportionate information tool for radiation alarm situations.

A solar particle event associated with a drastic increase in the $D$-index for a particular flight could be communicated to the cockpit crew for instance by ACARS (Aircraft Communications Addressing and Reporting System) and trigger both begin and end of a corresponding mitigation measure. The restriction of this measure to the period of significantly increased dose rates can reduce the economic impact and 
can be even a prerequisite for an application of such action, e.g. in case of fuel restrictions.

In order to establish a mitigation procedure involving immediate action it would be essential to develop a method for the real-time assessment of dose rates at aviation altitudes during a GLE. This could be, in general, realized either by a model framework in combination with real-time measurements of cosmic rays or high-quality on-board dose rate measurements.

\section{Radiation exposure in aviation}

Most of the time, the increased radiation exposure at aviation altitudes is solely caused by galactic cosmic rays (GCR) and secondary particles produced by interactions of the GCR with the atmosphere. The radiation exposure due to GCR varies slowly by less than a factor of 2 during the solar cycle and has, due to the large fraction of highly energetic particles, a moderate altitude and latitude dependence at commercial cruising altitudes between about $9 \mathrm{~km}$ and $12 \mathrm{~km}$ (EURADOS 2004).

The second component that may contribute to the radiation field is solar energetic particles accelerated during solar events which are typically related to strong solar X-ray flares and coronal mass ejections. In rare cases, on average about once a year, the increase in high-energy particle intensity is strong enough to be measured on ground by neutron monitors; such events are called ground level enhancements (GLE). Due to the steep slope in the energy distribution of the solar energetic particles, the dose rate in the atmosphere has a much stronger dependence on altitude and latitude than the galactic cosmic ray component.

The related radiation exposure is usually expressed in effective dose which is the sum over the organ doses weighted by the biological impact of different radiation types and the radio-sensitivity of the different organs. In this work the definition of the effective dose from ICRP (1991) and EU (1996) is used. For galactic cosmic rays typical values for intercontinental flights are on the order of several tens up to about one hundred micro-sievert $(\mu \mathrm{Sv})$, depending on the specific route and the period during the solar cycle. Estimations of the additional radiation exposure during ground level enhancements range from a few micro-sievert up to a few milli-sievert (e.g. Dyer et al. 2007; Copeland et al. 2008; Matthiä et al. 2009a, 2009b; Bütikofer \& Flückiger 2011).

The 70th event in the chronologically ordered list of ground level enhancements, which is the basis of this investigation, began in the early morning of 13th December 2006 following a solar X-ray flare on the western hemisphere of the sun $\left(5^{\circ} \mathrm{S}, 23^{\circ} \mathrm{W}\right)$. At 2:39 UTC, GOES-11 (Geostationary Operational Environmental Satellite) recorded the peak in solar $\mathrm{X}$-ray flux of $3.29 \times 10^{-4} \mathrm{~W} / \mathrm{cm}^{2}$ corresponding to an X3 class flare on the NOAA scale. Ten minutes later at around 2:50 UTC the energetic proton flux measured by GOES-11 started to increase and the integral flux of protons above $100 \mathrm{MeV}$ reached its maximum of $8.9 \times 10^{1} \mathrm{~cm}^{-2} \mathrm{~s}^{-1} \mathrm{sr}^{-1}$ between 5:25 UTC and 5:35 UTC. In contrast, the peak flux in the integral channel of protons above $10 \mathrm{MeV}$ of about $7 \times 10^{2} \mathrm{~cm}^{-2} \mathrm{~s}^{-1} \mathrm{sr}^{-1}$ was reached around $5 \mathrm{~h}$ later $(10: 30$ UTC) and corresponded to a NOAA S2 alert. Even though this is the second lowest solar radiation alert level ("moderate") on the NOAA $S$-scale, the event manifested as ground level enhancement in the measurements of many stations in the

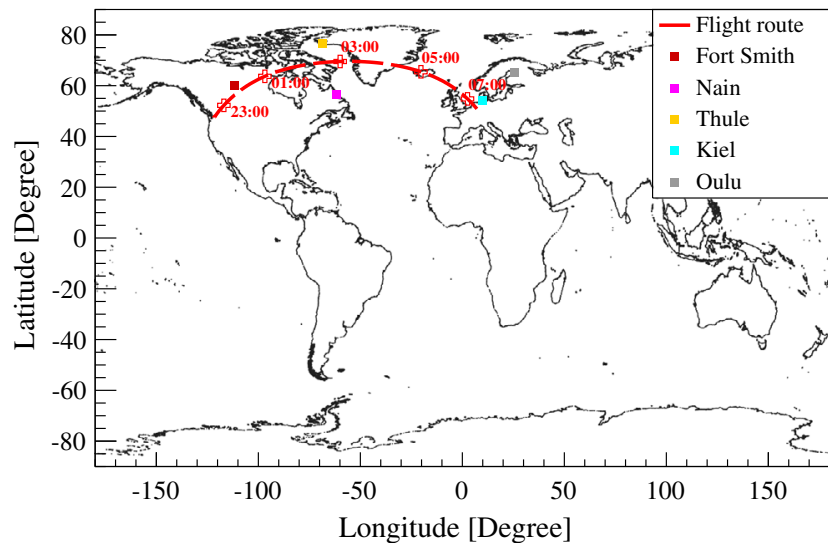

Fig. 1. Flight route from Seattle to Cologne (red dashed line; position of aircraft at different times UTC is indicated by the crosses) and the location of different neutron monitor stations close to the route (coloured squares).

neutron monitor network due to the relatively large fraction of highly energetic primary protons. The increase of the neutron monitor count rates and the integral proton channels commenced at about the same time. However, the shielding of the neutron monitors from cosmic rays at sea-level provided by the atmosphere means that the neutron monitors are mostly sensitive to the very highly energetic primary particles. Typically, sea-level neutron monitors at high latitudes are sensitive to protons with energies above approximately $500-1000 \mathrm{MeV}$ (e.g. Mishev et al. 2013). As the most energetic particles are produced only in the very beginning of an event and the intensities of the moderate and lower energetic particles tend to increase more slowly, the peaks in count rates were reached at different times: at around 3:00 UTC for the neutron monitors; at around 5:25 UTC for the integral proton flux above $100 \mathrm{MeV}$; and at around 10:30 UTC for the integral proton flux above $10 \mathrm{MeV}$. As the atmosphere still provides quite a strong shielding at aviation altitudes of about $200 \mathrm{~g} / \mathrm{cm}^{2}$ column mass density at $40,000 \mathrm{ft}$. (ca. 12,200 m), the peak in the radiation exposure is expected sometime between the peak in the neutron monitor count rates and the integral proton channel above $100 \mathrm{MeV}$.

Another typical phenomenon that was observed during the ground level enhancement was the anisotropy of the primary particle intensity, especially during the initial phase of the event. The intensity of primary particles on top of the atmosphere was distributed in a way that the neutron monitors that recorded the very prompt and strong increase were located at low eastern longitudes (cf. Figs. 1 and 2a). About 30 min after the onset of the event, the particle intensity became more equally distributed and neutron monitors at different locations measured similar count rate increases. In the same way, the increases in the radiation exposure from solar energetic particles at aviation altitudes are also expected to have been longitude dependent in the initial phase of the event (see also Matthiä et al. 2009b; Meier \& Matthiä 2014).

\section{Material and methods}

\subsection{PANDOCA}

PANDOCA (Matthiä et al. 2014) is a Monte-Carlo based model for the calculation of secondary particle fluxes induced by cosmic rays in the atmosphere and the related radiation 

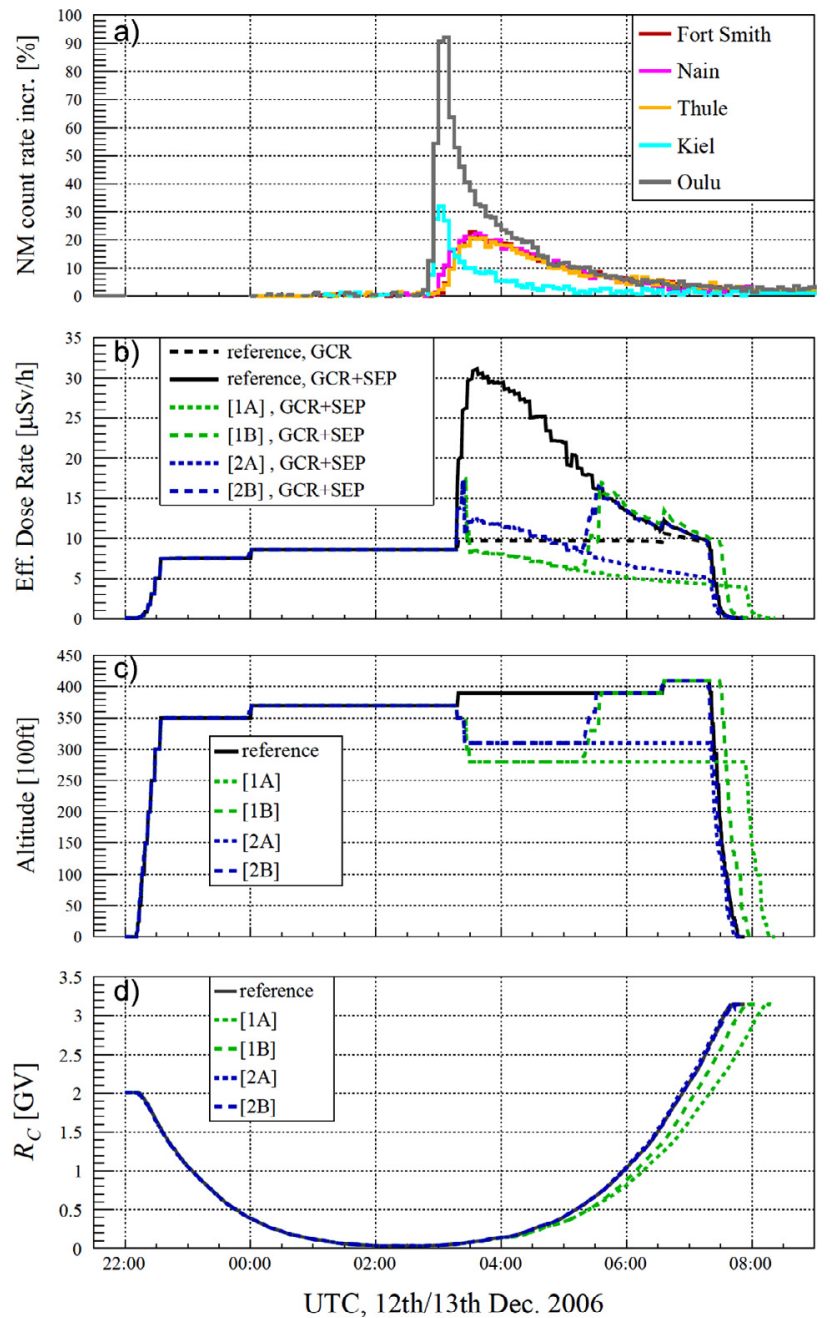

Fig. 2. From top to bottom: (a) relative count rate increases of selected neutron monitor stations located in proximity of the analysed flight route (cf. Fig. 1); (b) calculated effective dose rates from galactic cosmic rays (GCR) and solar energetic particles (SEP) for the different scenarios; (c) altitude profiles of the different flight scenarios; (d) effective cut-off rigidities $R_{\mathrm{c}}$ of the different scenarios.

exposure at aviation altitudes. In general, PANDOCA is capable of calculating the radiation environment for arbitrary primary spectra of cosmic ray nuclei at arbitrary points in the atmosphere and the associated dose rates. The structure and the composition of the atmosphere were taken from the NRLMSISE-00 model (Picone et al. 2002). For galactic cosmic ray particles PANDOCA has a built-in implementation of the galactic cosmic ray model by Matthiä et al. (2013). In addition, for solar cosmic rays in case of energetic particle events and ground level enhancements, the primary proton spectrum during the event can be added to the galactic cosmic ray spectrum. PANDOCA uses pre-calculated fluxes and dose rates for all relevant secondary particles at a large number of different altitudes. These fluxes and dose rates are scaled according to the primary input spectrum and, to calculate the quantities at a specific altitude, interpolated between the lower and upper neighbouring value in the list of pre-calculated altitudes.

The shielding from charged particles provided by the Earth's magnetosphere is considered on the basis of the effective vertical cut-off rigidity $R_{\mathrm{c}}$ (details on terminology can be found in Cooke et al. 1991). The cut-off rigidity in PANDOCA for a grid of geographical latitude and longitude was calculated with PLANETOCOSMICS (http://cosray.unibe.ch/ laurent/ planetocosmics/) using the international geomagnetic reference field (Maus et al. 2005). For each point on a flight route the cut-off rigidity is calculated and converted to cut-off energy for the primary particles. This cut-off energy is used as a lower threshold in the energy spectrum.

The ground level enhancement 70 was investigated and described in terms of temporal evolution and spatial distribution in Matthiä et al. (2009b). Here, we use the description of the primary spectra during the event to determine for each point on the flight route the corresponding primary input spectrum to be used in the calculation of the radiation exposure with PANDOCA.

\subsection{VarMission}

VarMission (Schaefer 2012) is aircraft performance software able to simulate flight missions of a variety of aircraft types in order to determine the flight trajectory, fuel consumption and gaseous emissions. The software can be used in combination with aircraft and engine models of different complexity including e.g. BADA (Base of Aircraft DAta) v3.x aircraft models (EUROCONTROL 2012) as well as tabulated performance data originating from aircraft manufacturers or design studies. Here, tabulated performance data providing an aircraft's specific range as function of gross weight, flight altitude and Mach number are used to simulate a generic medium-sized wide body aircraft similar in size and range to an Airbus A330200. While specific-range tables are used to simulate cruise flight, typical departure and approach procedures as well as climb and descent flight phases are simulated on the basis of the BADA model of the A330-200 (EUROCONTROL 2012). Characteristic aircraft weights (empty weight, maximum take-off weight, etc.) are also obtained from BADA.

In an iterative process, VarMission analyses each flight segment from taxi-out to taxi-in and determines the fuel burn along the flight profile. As fuel is consumed during the flight, aircraft mass is decreasing. Either the aircraft's payload or its initial take-off weight is required as input for a flight mission simulation. For simplicity, atmospheric conditions according to the International Standard Atmosphere (ISA) and no wind are assumed here when calculating fuel consumption. Typical reserve fuel policies similar to those used by airlines are assumed and include $5 \%$ of the trip fuel as contingency, additional fuel required to reach an alternate airport situated 200 nautical miles from the planned destination and holding fuel for an additional $30 \mathrm{~min}$ of holding.

\subsection{Flight profile selection}

\subsubsection{Reference scenario}

To quantify the effects of the ground level enhancement on radiation exposure in aviation a realistic flight scenario was identified. As the event peaked during the early morning hours (UTC) on December 13th 2006, the most affected flights would have been located at high latitudes with low geomagnetic shielding at this time. This is typically the case for passenger flights from the USA to Europe. A trajectory from Seattle to Cologne along the great circle was selected as representative for such a scenario (see Fig. 1). In order to maximize the theoretical impact on the radiation exposure during GLE70, 
Table 1. Flight scenarios for which the radiation exposure was calculated.

\begin{tabular}{ll}
\hline \hline Scenario & \multicolumn{1}{c}{ Description } \\
\hline Reference & Flight from Seattle to Cologne, starting at 22:00 UTC on December 12th 2006, flight duration $9 \mathrm{~h}, 54 \mathrm{~min}$, maximum altitude \\
& $41,000 \mathrm{ft}(\mathrm{ca} .12,500 \mathrm{~m})$.
\end{tabular}

different departure times were assessed for the same flight route and altitude profile. It was found that the maximum total effective dose for a flight from Seattle to Cologne during GLE70 would have occurred for a departure on 12th December at 22:00 UTC. Flight route, altitude profile and fuel consumption were determined using the VarMission aircraft performance software described in Section 3.2. A generic aircraft type similar to an Airbus A330-200 was assumed for this flight with a payload of 37 tons. This payload corresponds to a load factor of $75 \%$ and is equivalent to 270 passengers assuming a passenger weight of $100 \mathrm{~kg}$ including luggage plus 10 tons of cargo. Further assumptions include a long-range-cruise (LRC) Mach number in combination with a step-climb profile during cruise flight (see Fig. 2c). At LRC, the flight Mach number is chosen such that $1 \%$ of specific range is traded for higher flight speed, an assumption which is similar to current airline practice. Often airlines choose to fly slightly faster than the fueloptimal speed in order to reach minimum operating costs, which include time costs (Matthes et al. 2011). For the aircraft considered here the LRC Mach number during its cruise flight at near-optimal flight levels is between Mach 0.81 and 0.82 . As this scenario does not include any mitigating action it serves as a reference for comparison.

\subsubsection{Mitigation scenarios}

In addition to the reference scenario, four variations of this scenario were simulated aiming at a reduction of dose rates and radiation exposure resulting from the ground level enhancement (Table 1). While the aircraft's take-off mass is the same as for the reference flight, in the four alternative scenarios it is assumed that up to $5 \%$ of additional fuel consumption is acceptable for mitigation measures, as this amount is available on board as contingency fuel:

- Scenario [1A]: After the onset of the ground level enhancement and the related increase in radiation exposure, the flight level is reduced to the lowest altitude $(\mathrm{FL} 280 \triangleq 28,000 \mathrm{ft}$, ca. $8500 \mathrm{~m}$ ) for which the airplane can still reach its destination airport. When changing (reducing) cruise altitude, the flight Mach number is adapted (reduced) accordingly in order to maintain LRC flight. The reduced flight speed at reduced altitude results in increased flight duration. Scenario [1A] can be considered as a prompt response to an alert system indicating a higher radiation exposure of the plane due to the ground level enhancement at 3:15 UTC.
- Scenario [1B]: As scenario [1A], i.e. with a lowered flight altitude beginning with the onset of the event but returning to the optimized flight profile after the additional effective dose rate fell below a threshold of $10 \mu \mathrm{Sv} / \mathrm{h}$ which corresponds to a Space Weather index D1 or less (Meier \& Matthiä 2014). Scenario [1B] can be considered as a prompt response to an alert system indicating a higher radiation exposure on the plane and a corresponding cancellation of the radiation alarm.

- Scenario [2A]: As scenario [1A], but without adapting the Mach number. In contrast to [1A] the flight is not delayed in this scenario, but the possible altitude reduction is smaller.

- Scenario [2B]: As scenario [1B], but without adapting the Mach number. In contrast to [1B] the flight is not delayed in this scenario, but the possible altitude reduction is smaller.

\section{Results}

Figure 2 summarizes important parameters and results for the different flight scenarios. Figure $2 \mathrm{a}$ shows the relative count rate increases of different neutron monitor stations along the flight route. Below, the calculated effective dose rates for the four scenarios are illustrated (Fig. 2b). For the reference flight, both the total dose rates, i.e. the sum of galactic cosmic rays (GCR) and solar energetic particles (SEP), as well as the contribution of galactic cosmic rays alone are given. The changes in flight altitude (Fig. 2c) are reflected by corresponding changes in dose rate. Finally, Figure $2 d$ illustrates the geomagnetic shielding expressed by the effective vertical cut-off rigidity $R_{\mathrm{c}}$. Initially, during the early phase of the event, the plane is at a location of very low geomagnetic shielding and closest to the Nain and Thule stations. Accordingly, the increase in effective dose rate occurs simultaneously with the rise of count rates in these neutron monitors at around 3:15 UTC. The anisotropic angular distribution of the incoming primary particles is obvious from the observed count rates of the Oulu and Kiel stations that already had recorded elevated cosmic ray intensity some 20 min earlier. While the effective dose rate from galactic cosmic rays at that moment was calculated to have been on the order of $10 \mu \mathrm{Sv} / \mathrm{h}$ (long dashed line), the solar energetic particles added another $20 \mu \mathrm{Sv} / \mathrm{h}$ to a total of $30 \mu \mathrm{Sv} / \mathrm{h}$ peak dose rate for the reference flight route (solid line). An additional effective dose rate above $20 \mu \mathrm{Sv} / \mathrm{h}$ from solar energetic particles would have corresponded to a $D$-index of 3 . To mimic the response to an alarm system, the flight altitudes were lowered 
D. Matthiä et al.: Economic impact and effectiveness of radiation protection measures in aviation

Table 2. Calculated total effective dose, ambient dose equivalent, fuel consumption and flight duration for the different flight scenarios. Differences in percentage to the reference optimized flight are given in brackets. In case of doses, the relative differences are given with respect to the sum of galactic cosmic rays (GCR) and the ground level enhancement (GLE).

\begin{tabular}{lcccr}
\hline \hline Scenario & Effective dose $(\mu \mathrm{Sv})$ & Ambient dose equivalent $(\mu \mathrm{Sv})$ & Fuel consumption $(\mathrm{t})$ & Flight duration $(\mathrm{h})$ \\
\hline GCR (reference) & 80.2 & 67.2 & 53.9 & 9.90 \\
GCR + GLE (reference) & 119 & 112 & 53.9 & 9.90 \\
GCR + GLE [1A] & $68.7(-42 \%)$ & $62.7(-44 \%)$ & $56.5(+4.8 \%)$ & $10.37(+4.7 \%)$ \\
GCR + GLE [1B] & $83.6(-30 \%)$ & $74.3(-34 \%)$ & $55.2(+2.4 \%)$ & $10.06(+1.7 \%)$ \\
GCR + GLE [2A] & $75.2(-37 \%)$ & $69.3(-38 \%)$ & $56.6(+5.0 \%)$ & $9.83(-0.7 \%)$ \\
GCR + GLE [2B] & $87.3(-27 \%)$ & $78.9(-30 \%)$ & $55.2(+2.4 \%)$ & $9.86(-0.3 \%)$ \\
\hline
\end{tabular}

for all other scenarios shortly after the onset of the event to FL280 ( $\triangleq 28,000 \mathrm{ft}$., ca. $8500 \mathrm{~m})$ in case the Mach number was adapted (scenarios [1A] and [1B]; green lines) and to FL310 ( $\triangleq 31,000 \mathrm{ft}$. , ca. $9500 \mathrm{~m}$ ) in case the Mach number was not adapted (scenarios [2A] and [2B]; blue lines). In all cases the effective dose rate would have decreased significantly as a consequence of this action; in case of FL280 $(\approx 8.5 \mu \mathrm{Sv} / \mathrm{h})$ even below the galactic cosmic ray background of the reference flight $(\approx 9.5 \mu \mathrm{Sv} / \mathrm{h})$ which would have been at FL390 $(\triangleq 39,000 \mathrm{ft}$., ca. $12,000 \mathrm{~m})$ at that time. At around $4: 50$ UTC, the calculated effective dose rate at FL310 would have dropped below the GCR background of the reference flight as well. If such a prompt response had been performed and the flight altitude had been decreased in such a way, the additional effective dose rates would have been between $5 \mu \mathrm{Sv} / \mathrm{h}$ and $10 \mu \mathrm{Sv} / \mathrm{h}(D 1)$ in all mitigation scenarios.

For scenarios $[1 \mathrm{~B}]$ and $[2 \mathrm{~B}]$ a threshold for the additional effective dose rates from solar particles was defined below which the plane should return to the corresponding optimal flight altitudes. A threshold of $10 \mu \mathrm{Sv} / \mathrm{h}(D=1)$ was defined based on the calculated effective dose rate at $90^{\circ} \mathrm{N}$ at FL410 ( $\triangleq 41,000 \mathrm{ft}$., ca. $12,500 \mathrm{~m}$ ) which can be assumed as an upper limit if isotropy of the primary particles is assumed. The threshold corresponds to approximately a doubling of the dose rate from GCR and the calculated effective dose rate fell below this threshold at 5:18 UTC. Accordingly, in the corresponding scenarios [1B] and [2B], the airplane returned to the reference altitude (FL390) while in scenarios [1A] and [2A] the lower flight levels were maintained. Figure $2 \mathrm{~b}$ clearly shows that the effective dose rates for scenarios $[1 \mathrm{~B}]$ and $[2 \mathrm{~B}]$ would be similar to the dose rates of the reference flight after returning to higher altitudes while the dose rates for scenarios [1A] and [2A] stay well below the GCR contribution of the reference flight. The slight difference of the dose rates calculated for scenario [1B] in comparison to scenario [2B] and the reference flight pattern is related to the adapted Mach number which causes a delay in the flight. As a consequence the airplane would be in such a scenario at higher latitude at the same time and the magnetic shielding $\left(R_{\mathrm{c}}\right)$ would be lower (Fig. 2d). It can already be concluded from Figure $2 \mathrm{~b}$ that all four scenarios would have led to a significantly lower radiation exposure compared to the reference scenario.

The total effective doses and ambient dose equivalents for all cases are summarized in Table 2 together with the total fuel consumption and flight duration. The total effective dose for the reference flight profile from Seattle to Cologne during the ground level enhancement 70 was calculated to have been $119 \mu \mathrm{Sv}$, almost $70 \%(80 \mu \mathrm{Sv})$ of which were caused by galactic cosmic rays and their secondaries. All cases in which the flight altitude was lowered were calculated to have a significantly lower radiation exposure compared to the reference flight regarding the sum of galactic cosmic rays and the ground level enhancement (between $-27 \%$ and $-44 \%$ ). The maximum reduction in the effective dose that could be achieved by lowering the flight level within a limit of an additional fuel consumption of $5 \%$ is $-42 \%$ (case [1A]). While the effective dose is used for radiation protection purposes, the ambient dose equivalent $H^{*}(10)$ is experimentally accessible and an important part of measurement practice. Comparing the values of the ambient dose equivalent calculated by numerical models with experimental results is an important part of the validation of such models.

\section{Summary}

The possibility of prompt responses to ground level enhancements has been discussed within the scientific community and among pilots for many years. Little, however, is known about the feasibility and effectiveness of such responses concerning the reduction of the additional radiation exposure caused by solar energetic particles. For the first time we combine in this work the calculation of the radiation exposure with realistic flight and mitigation scenarios. For a representative flight from Seattle to Cologne during the ground level enhancement on December 13th 2006 it was found that it would have been possible to lower the flight altitude to such a degree that the total effective dose from solar and galactic cosmic rays would be reduced by up to $42 \%(50 \mu \mathrm{Sv})$ compared to the reference flight. This response could have been performed as a reaction to the increased Space Weather index $D$. In case such a response had been performed the $D$-index would have remained at $D 1$ rather than $D 3$ in case of no response.

The maximal reduction could have been achieved by an additional 5\% fuel expenditure and a flight delay of about $30 \mathrm{~min}$. For other scenarios without delay and/or less fuel expenditure the reduction of flight altitude that could be performed and the corresponding reduction in effective dose and ambient dose equivalent are lower. It has to be emphasized that in this work only the mitigating effect of altitude reduction has been investigated. Other possible measures could be a rerouting of flights to lower latitudes or changes in the flight schedule. It also has to be stated that such prompt changes in altitude and velocity as investigated in this work may be constrained by wind and weather conditions and are currently not compliant with the Air Traffic Management (ATM) System. In the future, however, improved radar coverage over the ocean through, for example, satellite-based receivers for Automatic Dependent Surveillance Broadcasts (ADS-B) may permit such operations (Delovski et al. 2014) and make it 
possible to reduce the radiation exposure of airline crew and passengers in case of ground level enhancements.

Acknowledgements. We thank the Bartol Research Institute for providing Thule, Nain and Fort Smith neutron monitor data, the Extraterrestrial Physics Department of the Institute for Experimental and Applied Physics of the Christian-Albrechts University of Kiel for providing Kiel neutron monitor data and the Sodankylä Geophysical Observatory for providing Oulu neutron monitor data (http:// cosmicrays.oulu.fi/). The Bartol Research Institute neutron monitor programme is supported by the United States National Science Foundation under Grants PLR-1245939 and PLR-1341562, and by the University of Delaware Department of Physics and Astronomy and Bartol Research Institute. Construction of the stations in Fort Smith, and Nain was supported by NSF's Major Research Infrastructure Program.

The editor thanks K. Copeland and an anonymous referee for their assistance in evaluating this paper.

\section{References}

Bütikofer, R., and E.O. Flückiger. Radiation doses along selected flight profiles during two extreme solar cosmic ray events. Astrophys. Space Sci. Trans., 7, 105-107, 2011,

DOI: 10.5194 /astra-7-105-2011.

Cooke, D.J., J.E. Humble, M.A. Shea, D.F. Smart, N. Lund, I.L. Rasmussen, B. Byrnak, P. Goret, and N. Petrou. On cosmic-ray cut-off terminology. Il Nuovo Cimento C, 14 (3), 213-234, 1991, DOI: $10.1007 / B F 02509357$.

Copeland, K., H.H. Sauer, F.E. Duke, and W. Friedberg. Cosmic radiation exposure of aircraft occupants on simulated highlatitude flights during solar proton events from 1 January 1986 through 1 January 2008. Adv. Space Res., 42, 1008-1029, 2008, DOI: $10.1016 /$ j.asr.2008.03.001.

Delovski, T., K. Werner, T. Rawlik, J. Behrens, J. Bredemeyer, and R. Wendel. ADS-B over satellite the world's first ADS-B receiver in Space, Proceedings of the Small Satellites Systems \& Services Symposium 2014 - The 4S Symposium, Porto Petro, Majorca, Spain, 2014.

Dyer, C., F. Lei, A. Hands, and P. Truscott. Solar particle events in the QinetiQ atmospheric radiation model. IEEE Trans. Nucl. Sci., 54 (4), 1071-1075, 2007, DOI: 10.1109/TNS.2007.893537.

EU. Council Directive 96/29/EURATOM. Official Journal of the European Communities, N ${ }^{\circ}$ L 159 of 29.6.96, 1996.

EURADOS. Cosmic radiation exposure of aircraft crew. European Radiation Dosimetry Group report, ISBN: 92-894-8448-9, 2004, Braunschweig, Germany.
EUROCONTROL. User manual for the Base of Aircraft Data (BADA) revision 3.10. EEC Technical/Scientific Report No. 12/04/10-45, 2012.

ICRP. 1990 Recommendations of the International Commission on Radiological Protection. Annals of the ICRP, 21 (1-3), 1-201, 1991.

ICRP. The 2007 Recommendations of the International Commission on Radiological Protection. Annals of the ICRP, 37 (2-4), 1-332, 2007.

Matthes, S., K. Gierens, V. Grewe, H. Mannstein, R. Sausen, V. Mollwitz, M. Schaefer, and M. Weiss. Climate optimized flight planning. Abschlussbericht zum BMU-Vorhaben 300/2008, 2011.

Matthiä, D., B. Heber, G. Reitz, M. Meier, L. Sihver, T. Berger, and K. Herbst. Temporal and spatial evolution of the solar energetic particle event on 20 January 2005 and resulting radiation doses in aviation. J. Geophys. Res., 114, A08104, 2009a, DOI: $10.1029 / 2009 J A 014125$.

Matthiä, D., B. Heber, G. Reitz, L. Sihver, T. Berger, and M. Meier. The ground level event 70 on December 13th, 2006 and related effective doses at aviation altitudes. Radiat. Prot. Dosim., 136 (4), 304-310, 2009b, DOI: 10.1093/rpd/ncp141.

Matthiä, D., T. Berger, A.I. Mrigakshi, and G. Reitz. A ready-to-use galactic cosmic ray model. Adv. Space Res., 51, 329-338, 2013, DOI: $10.1016 /$ j.asr.2012.09.022.

Matthiä, D., M.M. Meier, and G. Reitz. Numerical calculation of the radiation exposure from galactic cosmic rays at aviation altitudes with the PANDOCA core model. Space Weather, 12, 161-171, 2014, DOI: 10.1002/2013SW001022.

Maus, S., S. Macmillan, T. Chernova, S. Choi, D. Dater, et al. The 10th-generation international geomagnetic reference field. Geophys. J. Int., 161, 561-565, 2005, DOI: 10.1029/2005EO160006.

Meier, M.M., M. Hubiak, D. Matthiä, M. Wirtz, and G. Reitz. Dosimetry at aviation altitudes (2006-2008). Radiat. Prot. Dosim., 136, 4, 251-255, 2009, DOI: 10.1093/rpd/ncp142.

Meier, M.M., and D. Matthiä. A space weather index for the radiation field at aviation altitudes. J. Space Weather Space Clim., 4, A13, 2014, DOI: 10.1051/swsc/2014010.

Mishev, A.L., I.G. Usoskin, and G.A. Kovaltsov. Neutron monitor yield function: new improved computations. J. Geophys. Res. [Space Phys.], 118 (6), 2783-2788, 2013.

Picone, J.M., A.E. Hedin, and D.P. Drob. NRLMSISE-00 empirical model of the atmosphere: statistical comparisons and scientific issues. J. Geophys. Res., 107 (A12), 1468, 2002, DOI: $10.1029 / 2002 J A 009430$.

Schaefer, M. Development of a forecast model for global air traffic emissions, DLR Forschungsbericht 2012-08, 2012.

Cite this article as: Matthiä D, Schaefer M \& Meier MM. Economic impact and effectiveness of radiation protection measures in aviation during a ground level enhancement. J. Space Weather Space Clim., 5, A17, 2015, DOI: 10.1051/swsc/2015014. 